\title{
Chirurgische Konzepte und Strategien bei Kolonadenomen und Polyposissyndromen
}

\author{
S. Pistorius U. Wehrmann E.-M. Teichert H.-D. Saeger \\ Klinik und Poliklinik für Viszeral-, Thorax- und Gefäßchirurgie, Universitätsklinikum Carl Gustav Carus, TU Dresden
}

\section{Schlüsselwörter \\ Kolorektales Karzinom: hereditäre Formen, Prävention \\ Überwachung, chirurgisches Vorgehen}

\section{Zusammenfassung}

Die Entwicklung moderner minimal-invasiver Diagnose- und Behandlungsverfahren auf dem Gebiet der kolorektalen Adenome und Karzinome ermöglicht eine effektive Überwachung von Risikopersonen, andererseits erfolgt durch die endoskopische Abtragung oder transanale bzw. TEM-technische Resektion von Adenomen bereits eine erhebliche Karzinomprävention. Die Einführung der laparoskopischen Technik bei der Resektion kolorektaler Tumoren könnte nach Evaluierung der bisherigen Ergebnisse zu einer weiteren Verringerung der Hospitalisierung und operationsassoziierten Morbidität der Patienten bei gleicher Prognose führen. Kennzeichnend für familiäre Formen des kolorektalen Karzinoms ist das hohe Risiko für die Entwicklung kolorektaler Tumoren, jedoch auch für weitere extrakolonische Neoplasien. Dies trifft für das hereditäre Nicht-Polyposis-assoziierte kolorektale Karzinom (HNPCC), die familiäre Polyposis (FAP) und die selteneren Formen wie Peutz-Jeghers-Syndrom und juvenile Polyposis zu. Die Anwendung der molekularen Diagnostik in diesen Familien ermöglicht durch die Identifizierung von Mutationsträgern und Nichtmutationsträgern einerseits die gezielte Eingliederung von Hochrisikopersonen (Mutationsträgern) in spezielle, auf das jeweilige Syndrom zugeschnittene Überwachungs- und Vorsorgeprogramme und erspart andererseits Personen mit durchschnittlichem Risiko (Nichtmutationsträgern) unnötige und teilweise invasive Diagnostik. Bezüglich des chirurgischen Vorgehens bei Patienten mit einer Form des hereditären kolorektalen Karzinoms gibt es bereits etablierte Verfahren, wie die Durchführung einer restaurativen Proktokolektomie bei der FAP, bei anderen Formen, wie bei HNPCC, sind diese noch in der Diskussion. Wesentliche Fortschritte bei der Prävention kolorektaler Tumoren sind in den nächsten Jahren möglicherweise auf dem Gebiet der Chemoprävention zu erwarten.

\section{Key Words}

Colorectal cancer: hereditary forms, prevention, surveillance, surgical approach

\section{Summary}

Surgical Approach and Strategies in Colon Adenomas and Polyposis Syndromes

Development of modern, minimally invasive methods for diagnosis and treatment in the field of colorectal tumours enables an effective surveillance for persons at high risk as well as a distinct cancer prevention by endoscopic, transanal or TEM removal of colorectal adenomas. Introduction of laparoscopic techniques in the resection of colorectal tumours could entail, after evaluation of preliminary results, a decreased duration of hospitalisation and procedure-associated morbidity in patients with the same prognosis. The very high risk for development of colorectal tumours as well as for some extracolonic neoplasia is typical for familial colorectal cancer syndromes. This concerns hereditary nonpolyposis colorectal cancer (HNPCC) syndrome, familial polyposis coli, and the infrequent forms like Peutz-Jeghers syndrome and juvenile polyposis. Molecular diagnostics has the power to identify carriers and noncarriers of a mutated gene in these families and therefore may permit clear-cut decisions regarding inclusion in special surveillance programmes, which is recommended for all persons at risk from affected families. Concerning the surgical approach in patients with hereditary colorectal cancer, well-accepted routine procedures like restorative proctocolectomy in familiar polyposis patients have already been established; in other forms like HNPCC the best surgical modality is still under discussion. Remarkable progress in the prevention of colorectal tumours could be expected from chemoprevention trials in the next years.

\begin{tabular}{ll}
\hline KARGER & @ 2002 S. Karger GmbH, Freiburg \\
Fax +49 7614520714 & Accessible online at: \\
$\begin{array}{l}\text { E-mail Information@Karger.de } \\
\text { www.karger.com }\end{array}$ & www.karger.com/journals/cga
\end{tabular}




\section{Einleitung}

Kolorektale Karzinome sind das Ergebnis einer Karzinogenese, die mit kleinen Läsionen in Kolonkrypten beginnt, sich über Adenome verschiedener Dysplasiegrade fortsetzt und schließlich in einem manifesten Karzinom resultiert. Diese Abfolge klinischer und pathohistologischer Stadien wird als Adenom-Karzinom-Sequenz bezeichnet. Auf molekularer Ebene ist diese Sequenz mit der Akkumulation von Mutationen in verschiedenen Tumorsuppressor- und Onkogenen assoziiert, die für das Gleichgewicht zwischen Zellproliferation und Apoptose verantwortlich sind. Jede dieser Mutationen bewirkt einen Wachstumsvorteil des jeweiligen Zellklons in dem sich entwickelnden Tumor [1].

Bei den meisten Patienten umfasst der Zeitraum von der Entstehung des kolorektalen Adenoms bis zur Entwicklung des Karzinoms bis zu 10 Jahren. In bestimmten Fällen, wie z.B. bei Patienten mit dem «hereditary nonpolyposis colorectal cancer»(HNPCC)-Syndrom ist diese Zeit erheblich verkürzt, da es zu einer deutlichen Beschleunigung der Adenom-Karzinom-Sequenz kommt [2, 3].

Bei einer weiteren Gruppe von Patienten bedingen Keimbahnmutationen in Genen, die eine Rolle bei der Tumorinitiation spielen (Gatekeeper-Gene), das früh- bzw. massenhafte Auftreten kolorektaler Adenome. Dies betrifft z.B. Patienten mit familiärer adenomatöser Polyposis coli (FAP) [4].

Die in den letzten Jahren gewonnenen molekularbiologischen Erkenntnisse helfen bei dem Verständnis zur Entwicklung der verschiedenen Formen der kolorektalen Adenome und Karzinome, ermöglichen darüber hinaus jedoch neben der Option der prädiktiven molekularen Diagnostik und der damit verbundenen gezielten Vorsorge in Familien mit hereditären Formen eine prognostische Einschätzung des klinischen Verlaufs und die sich daraus ergebenden Konsequenzen des chirurgischen Handelns.

\section{Minimal-invasive chirurgische Therapie kolorektaler Adenome}

\section{Endoskopische Verfahren}

Die Ileokoloskopie als sensitivstes Verfahren für die Diagnostik kolorektaler Polypen und Karzinome ist auch in Kombination mit einer endoskopischen Polypektomie ein ausgesprochen sicheres und komplikationsarmes Untersuchungsverfahren. So wird die Häufigkeit einer Blutung mit $2 \%$ und die einer Perforation mit 0,3\% angegeben [5,6]. Die endoskopische Polypektomie gilt heute als das Standardverfahren für die Behandlung der meisten kolorektalen Polypen. Kleinere Polypen $(<5 \mathrm{~mm})$ werden mit der Zange und größere $(>5 \mathrm{~mm})$ mit der Schlinge in toto abgetragen. In Abhängigkeit von der Erfahrung des Endoskopikers können auch große, breitbasige Polypen des Kolons und des Rektums durch eine Mukosektomie nach submuköser Injektion einer Kochsalzlösung oder einer verdünnten Adrenalinlösung (1:100 000) mit der Diathermieschlinge abgetragen werden. Die einzeln geborgenen Polypen werden unter Angabe der Lokalisation histologisch befundet. Auf der Basis des histologischen Befundes werden die weitere Vorgehensweise und die Nachsorge festgelegt.

Im Zeitraum von 1991 bis 2001 wurden an der Klinik für Viszeral-, Thorax- und Gefäßchirurgie am Universitätsklinikum Carl Gustav Carus an der Technischen Universität Dresden bei 15217 Patienten Koloskopien durchgeführt. Dabei wurden bei 1595 Patienten 2871 endoskopische Polypektomien vorgenommen. 2240 Polypen (78\%) hatten einen Durchmesser von $<1 \mathrm{~cm}$ und 631 Polypen $(22 \%)$ von $>1 \mathrm{~cm}$. Bei $14 \mathrm{~Pa}-$ tienten $(0,88 \%)$ fand sich bei der histologischen Aufarbeitung ein Karzinom. In der Literatur wird die Häufigkeit eines Karzinoms in endoskopisch abgetragenen Adenomen mit 0,4-5\% angegeben [7-9]. Das Risiko eines Karzinoms in einem Adenom variiert mit der Größe und der anatomischen Lokalisation des Polypen und kann bei einem Polypen von $>2,5 \mathrm{~cm}$ Durchmesser im Rektum mehr als $50 \%$ betragen [8]. Bei 12 Patienten $(0,75 \%)$ trat bei der endoskopischen Polypektomie eine Blutung auf, die bei 10 Patienten endoskopisch therapiert werden konnte. Bei 2 Patienten war eine operative Versorgung erforderlich. Eine Perforation wurde bei 6 Patienten $(0,38 \%)$ mit einem Polypen $>1 \mathrm{~cm}$ beobachtet. Drei Patienten wurden bereits innerhalb $1 \mathrm{~h}$ nach Polypektomie mit Übernähung der Perforation operiert. Ein protektives Ileostoma konnte bei diesen Patienten vermieden werden.

Bei erhöhtem Perforationsrisiko ist zu empfehlen, in Abhängigkeit von Größe, Polypenform, Dignität, Lokalisation und dem Allgemeinzustand des Patienten im Einzelfall alternative Therapieverfahren wie die laparoskopische oder konventionelle Resektion, die laparoskopisch assistierte endoskopische Polypektomie oder die transanale endoskopische Mikrochirurgie einzusetzen.

\section{Minimal-invasive Verfahren}

Die letzten Jahre haben in der Chirurgie einen deutlichen Trend hin zu verschiedenen minival-invasiven Verfahren erkennen lassen. Voraussetzungen dafür waren einerseits die Entwicklung geeigneter Instrumentarien, andererseits die Generierung von Daten innerhalb verschiedener Studien, die einen Vorteil dieser Verfahren bezüglich Lebensqualität, stationärer Verweildauer, Mortalität und Morbidität bei gleicher Prognose und Rezidivrate sowohl bei Adenomen als auch kleinen Karzinomen belegen konnten. Dies trifft insbesondere für endoskopisch nicht abtragbare Rektumadenome und frühe Rektumkarziome zu. Durch die Einführung der transanalen lokalen Exzision bei tiefsitzenden $(0-5 \mathrm{~cm})$ und der transanalen endoskopischen mikrochirurgischen Resektion (TEM) bei höher sitzenden $(5-16 \mathrm{~cm})$ Rektumtumoren ist es möglich, einer Reihe von Patienten die konventionelle anteriore Rektumresektion bzw. abdominoperineale Rektumexstirpation zu ersparen. Voraussetzung für den Einsatz dieser beiden Verfahren ist ein exaktes präoperatives Staging, da 
Tab. 1. Formen der hereditären kolorektalen Karzinome und deren asssoziierte Gene [34, 65-82]

\begin{tabular}{ll}
\hline Syndrom & Gene \\
\hline FAP & APC \\
Peutz-Jeghers-Syndrom & STK11 \\
Juvenile Polyposis & $S M A D 4$, BMPR1A \\
HNPCC & $h M L H 1, h M L S H 2, h M S H 6$, PMS1, PMS2, \\
& EXO1 \\
\hline
\end{tabular}

nach den bisherigen Daten nur bei Rektumadenomen und -karzinomen im Stadium T1N0G1-2 die Indikation zu dieser Technik gegeben ist. In mehreren Studien konnte dabei gezeigt werden, dass Rezidivrate und Prognose dieser Patienten durch den Einsatz der minimal-invasiven Technik nicht negativ beeinflusst wurde [10-13].

Im Zeitraum von 1994 bis 2001 wurde in der Klinik für Viszeral-, Thorax- und Gefäßchirurgie des Universitätsklinikums Carl Gustav Carus Dresden bei 212 Patienten eine TEM-technische Resektion vorgenommen. Die Indikation dazu stellten in 168 Fällen Adenome und in 44 Fällen Karzinome dar. Von diesen 44 Patienten mit Rektumkarzinom erfolgte die Resektion in 35 Fällen mit kurativer Zielsetzung, wobei bei allen $\mathrm{Pa}-$ tienten eine R0-Resektion erzielt werden konnte. In 32 Fällen bestätigte sich histologisch das präoperativ diagnostizierte Tumorstadium T1N0G1-2. Lediglich bei 3 Patienten fand sich histologisch ein T2N0G1-2-Stadium, so dass bei diesen Patienten danach eine konventionelle, kontinenzerhaltende Resektion erfolgte; in keinem Fall fand sich dabei Restkarzinomgewebe im Präparat. Bei 9 der 44 Patienten mit Rektumkarzinom erfolgte die TEM-technische Resektion aufgrund allgemeiner Kontraindikationen gegen eine konventionelle Resektion unter palliativer Zielsetzung, wobei sich histologisch in den Präparaten bei 2 Patienten T2G1-, bei 4 Patienten T2G2- und bei 3 Patienten T3G2-Karzinome fanden.

Laparoskopische Resektionen bei benignen und malignen kolorektalen Tumoren erlangen eine immer größere praktische Relevanz. Hierbei ist es möglich, die für laparoskopische Resektionen bekannten Vorteile der minimalen Invasivität mit der Forderung nach Resektion entsprechend den onkologischen Resektionsprinzipien zu verbinden. In vorliegenden Multizenterstudien wurden die Überlebensrate und die Rezidivfreiheit durch die laparoskopische Technik nicht negativ beeinflusst $[14,15]$. Die bisher publizierten Ergebnisse bezüglich der Rate von perioperativen Komplikationen bzw. Anastomoseninsuffizienzen sind denen konventioneller Verfahren durchaus vergleichbar $[16,17]$.

\section{«Sporadische» kolorektale Adenome}

85-90\% aller kolorektalen Adenome gelten nach dem üblichen Verständnis als «sporadisch», da sich keine eindeutige
Familienanamnese findet oder keine Keimbahnmutation in einem der mit den hereditären Formen assoziierten Gene bei diesen Patienten bekannt ist. Dies bedeutet jedoch nicht, dass nicht eine Neumutation oder ein risikoassoziierter Polymorphismus in solchen oder anderen Genen, wie z.B. Genen des Karzinogenstoffwechsels [18], vorliegt und somit das individuelle kumulative Tumorrisiko deutlich erhöht oder der klinische Verlauf different oder beschleunigt ist. Ein Hinweis dafür kann z.B. die Entwicklung extrakolonischer, syndromassoziierter Manifestationen oder ein junges Erkrankungsalter sein.

\section{Hereditäre Kolonkarzinome und Polyposissyndrome}

Etwa 7\% aller kolorektalen Karzinome werden durch hereditäre Formen bedingt. Die häufigste Form mit etwa 5\% ist dabei das hepatozelluläre Nicht-Polyposis-assoziierte Kolonkarzinom(HNPCC)-Syndrom. Die familiäre Polyposis (FAP) mit etwa $1 \%$ ist deutlich seltener, juvenile Polyposis und Peutz-Jeghers-Syndrom können als sehr selten eingeschätzt werden. Ursache dieser autosomal-dominant vererbten Formen des kolorektalen Karzinoms sind hochpenetrante Keimbahnmutationen in bestimmten Genen (Tab. 1). Mutationsträger in diesen Genen haben ein deutlich erhöhtes Risiko für die Entwicklung kolorektaler Adenome bzw. Karzinome und weiterer syndromspezifischer extrakolonischer Manifestationen (s. unten).

\section{Molekulare prädiktive Diagnostik - Konsequenzen für die klinische Vorsorge}

Die molekulare Analyse der syndromassoziierten Gene bei Indexpatienten in Familien mit einer hereditären Form des kolorektalen Karzinoms ermöglicht die Identifizierung der jeweiligen pathogenen Keimbahnmutation. Danach sollte Risikopersonen der Familie die prädiktive molekulare Diagnostik angeboten werden. Während Nichtmutationsträger keiner speziellen Vorsorge bedürfen, müssen Mutationsträger in ein engmaschiges Vorsorgeprogramm, das auf das jeweilige familiäre Tumorsyndrom zugeschnitten ist (s. unten), eingebunden werden. Diese regelmäßige Vorsorge muss auch bei allen Risikopersonen in den Familien durchgeführt werden, in denen der Nachweis der pathogenen Keimbahnmutation nicht gelingt, die jedoch die entsprechenden Kriterien für das Vorliegen einer familiären Form des kolorektalen Karzinoms (s. unten) erfüllen.

Seit 1999 fördert die Deutsche Krebshilfe 6 Zentren für Krebsvorsorge und Krebsfrüherkennung bei erblichem kolorektalem Karzinom: Bochum, Bonn, Heidelberg, München, Dresden und Düsseldorf. Ziel dieses Pilotprojekts ist die Evaluierung der interdisziplinären Zusammenarbeit zwischen Molekularbiologen, Genetikern, Psychologen, Gastroenterologen und Chirurgen bei der Beratung, Diagnostik und Behandlung dieser Patienten. 


\section{Chemoprävention}

Die Wirksamkeit einer gezielten Chemoprävention bei Personen mit hohem Risiko für die Entwicklung kolorektaler Adenome und Karzinome, insbesondere bei Patienten mit hereditärer Form des kolorektalen Karzinoms konnte durch erste experimentelle und klinische Studien gezeigt werden. Die größte Bedeutung besitzen dabei COX2-Inhibitoren und Acetylsalicylsäure [19-21]. Acetylsalicylsäure scheint dabei insbesondere bei kolorektalen Tumoren mit bestimmtem genetischem Status (Mikrosatelliteninstabilität) zu einer Induktion von Apoptose zu führen [21]. Bei Patienten mit FAP und HNPCC, die ein stark erhöhtes Risiko für die Entwicklung kolorektaler Adenome und Karzinome besitzen, erfolgt gegenwärtig die Evaluierung dieser ersten Ergebnisse im Rahmen der klinischen CAPP-Studien. In deren Ergebnissen könnten einerseits Empfehlungen bezüglich einer gezielten Chemoprävention bei Hochrisikopersonen stehen, andererseits könnte dies das operative Vorgehen hinsichtlich prophylaktischer Eingriffe bei diesen Patienten beeinflussen.

In einer populationsbasierten Kohortenstudie [22] wurde eine Reduktion des relativen Karzinomrisikos auf 0,6 bei einer Langzeiteinnahme von $300 \mathrm{mg}$ Aspirin pro Tag beobachtet. Nichtsteroidale Antiphlogistika führten zu einer Halbierung des relativen Risikos in einem Zeitraum zwischen 6 Monaten nach Einnahmebeginn und 1 Jahr nach Beendigung der Einnahme.

\section{Familiäre adenomatöse Polyposis coli}

Die FAP ist durch das Auftreten von Hunderten von adenomatösen Polypen im Kolon und Rektum charakterisiert. Die kumulative Wahrscheinlichkeit für die Malignisierung mindestens eines dieser Polypen beträgt fast $100 \%$. Neben dem Auftreten verschiedener extrakolonischer Manifestationen (Desmoide, CHRPE u.a.) ist eine typische Genotyp-PhänotypKorrelation bekannt [23]: Während Keimbahnmutationen im zentralen Teil des $A P C$-Gens zur Ausbildung eines schweren Phänotyps mit frühzeitiger Karzinomentstehung führen [24], resultiert bei Keimbahnmutation am Anfang oder Ende des APC-Gens eine attenuierte Form (AFAP) mit deutlich weniger Polypen und späterem Manifestationsalter [25].

Das Überwachungsprogramm beinhaltet eine jährliche Rektosigmoidoskopie und Abdomensonographie ab dem 12. Lebensjahr, bei Beobachtung erster Polypen eine Koloskopie bis zur entsprechenden Operation. Darüber hinaus sollte eine regelmäßige Gastroduodenoskopie alle 3 Jahre, beim Nachweis von Adenomen jährlich ab dem 30. Lebensjahr bzw. vor einer Proktokolektomie erfolgen (Richtlinien des Verbundprojektes «Familiärer Darmkrebs» der Deutschen Krebshilfe) [26-28].

Ziel der Operation ist die Entfernung des gesamten Kolons und Rektums vor Entstehung eines Karzinoms. Chirurgischer Standard bei Patienten mit einer klassischen Form der FAP ist daher die Durchführung einer restaurativen Proktokolektomie $[29,30]$, wobei die Operation meist im frühen Erwach- senenalter indiziert ist. Die Frage, ob bei Patienten mit einer attenuierten FAP eine Kolektomie mit Ileorektostomie als entsprechender Eingriff durchgeführt werden kann, ist gegenwärtig noch nicht abschließend zu beantworten [31].

\section{Peutz-Jeghers-Syndrom}

Patienten mit einem Peutz-Jeghers-Syndrom entwickeln hamartomatöse Polypen im gesamten Gastrointestinaltrakt, die meisten davon im Dünndarm, wo sie durch Invagination des polypentragenden Darmabschnittes zu einem akuten Abdomen führen können [32]. Polypen treten auch häufig im Dickdarm und Magen auf. Peutz-Jeghers-Polypen zeigen gelegentlich auch einen adenomatösen Anteil, so dass auch die Theorie der Hamartom-Adenom-Karzinom-Sequenz diskutiert wird $[33,34]$. Typisch sind außerdem häufig bereits in den ersten Lebensjahren Pigmentflecken auf den Lippen, der Mundschleimhaut und perioral [35]. Patienten mit Peutz-JeghersSyndrom haben ein deutlich erhöhtes relatives Risiko für gastrointestinale und extraintestinale Karzinome, zusätzlich zu dem Risiko für kolorektale Karzinome und Magenkarzinome für Malignome der Mamma, der Cervix uteri, der Ovarien, des Pankreas, des Dünndarm und der Lunge [36-38].

Überwachungsprogramme für Patienten und Mutationsträger sollten eine 2-jährliche Gastroskopie und Koloskopie sowie regelmäßige gynäkologische Untersuchungen einschließlich Mammographie beinhalten (Richtlinien des Verbundprojekts «Familiärer Darmkrebs» der Deutschen Krebshilfe) [27, 35, 39].

Die Behandlung von Patienten mit Peutz-Jeghers-Syndrom konzentriert sich auf die Entfernung der Polypen. Dies ist bei Lokalisation der Polypen im Magen und Kolorektum meist unproblematisch endoskopisch möglich. Dünndarmpolypen sind jedoch auf diese Weise in der Regel nicht zu sanieren, andererseits führen wiederholte Dünndarmsegmentresektionen zu einem Kurzdarmsyndrom. Eine Alternative stellt die kombinierte chirurgisch-endoskopische Behandlung dar, bei der nach Laparotomie über eine Jejunotomie oder Ileotomie die endoskopische Polypektomie erfolgen kann [27, 40-42]. Tritt ein Karzinom auf, muss entsprechend den onkologischen Resektionsprinzipien vorgegangen werden.

\section{Juvenile Polyposis}

Patienten mit juveniler Polyposis entwickeln kolorektale Polypen, insbesondere im Rektum, die sich klinisch mit chronischen gastrointestinalen Blutungen und Anämie sowie einer damit verbundenen Entwicklungsverzögerung der Kinder manifestieren. Die exakte Diagnose wird histologisch gestellt. Juvenile Polypen können jedoch auch in adenomatöse Polypen übergehen. Folgende Kriterien wurden für die Diagnose einer juvenilen Polyposis vorgeschlagen: 1. >5-10 juvenile Polypen im Kolon, 2. juvenile Polypen im gesamten Gastrointestinaltrakt, 3. jegliche Anzahl juveniler Polypen bei einem Patienten mit Familienanamnese einer juvenilen Polyposis [43]. Bei Patienten mit familiärer juveniler Polyposis liegt das Risiko, 
Tab. 2. Amsterdam-Kriterien für HNPCC [83, 84]

\section{Amsterdam-I-Kriterien \\ Es sind mindestens 3 Familienangehörige mit histologisch gesichertem kolorektalem Karzinom betroffen, wobei 1 Angehöriger mit den beiden anderen erstgradig verwandt sein muss. Eine FAP muss ausgeschlossen sein. \\ Es sind wenigstens 2 aufeinander folgende Generationen betroffen. \\ Bei mindestens 1 Patienten erfolgte die Diagnosestellung vor dem 50. Lebensjahr.}

\section{Amsterdam-I-Kriterien}

Es sind mindestens 3 Familienangehörige mit histologisch gesichertem kolorektalem Karzinom oder einem Karzinom des Endometriums, Dünndarms oder Urothels (ableitende Harnwege/Nierenbecken) betroffen, wobei 1 Angehöriger mit den beiden anderen erstgradig verwandt sein muss. Eine FAP muss ausgeschlossen sein.

Es sind mindestens 2 aufeinander folgende Generationen betroffen.

Bei mindestens 1 Patienten erfolgte die Diagnosestellung vor dem 50. Lebensjahr.

bis zum 60. Lebensjahr an einem kolorektalen Karzinom zu erkranken, bei $20-60 \%$. Ferner besteht ein erhöhtes Risiko für Magen- und Duodenalkarzinome [44-46].

Vorsorgeempfehlungen sehen regelmäßige Koloskopien und Gastroduodenoskopien vor [27]. Bezüglich des Beginns der Untersuchungen und der Intervalle gibt es jedoch ebenso wenig Konsens wie zu Empfehlungen zum chirurgischen Management. Singuläre Polypen werden endoskopisch abgetragen, Karzinome entsprechend den onkologischen Resektionsprinzipien reseziert. Es wird jedoch auch die Durchführung einer restaurativen Proktokolektomie als optionale Erweiterung des Eingriffs im Fall einer notwendigen Kolonteilresektion diskutiert [47].

\section{Hereditäres Nicht-Polyposis-assoziiertes kolorektales \\ Karzinom}

Das HNPCC-Syndrom ist klinisch durch die Amsterdam-Kriterien I/II definiert (Tab. 2). HNPCC-Mutationsträger entwickeln meist frühzeitig kolorektale Karzinome (kumulatives Risiko etwa $80 \%$ ), insbesondere synchrone und metachrone kolorektale Karzinome, jedoch auch extrakolonische Karzinome, insbesondere des Endometriums, des Dünndarms, der ableitenden Harnwege und weiterer Organe [48, 49-52].

Das HNPCC-Vorsorgeprogramm umfasst folgende Untersuchungen: Koloskopie, gynäkologische Untersuchung einschließlich endovaginaler Sonographie, Abdomensonographie, urinzytologische Untersuchung und in Familien mit gehäuften Magenkarzinomen Gastroskopie. Dies sollte jährlich ab dem 25. Lebensjahr bzw. 5 Jahre vor Erstmanifestation eines Karzinoms in der Familie erfolgen (Richtlinien des Verbundprojekts «Familiärer Darmkrebs» der Deutschen Krebshilfe) $[27,53]$.

\section{Eingriffe bei Adenomen}

Regelmäßige Koloskopien mit Polypektomien bei Angehörigen von Familien mit HNPCC in Abständen von 3 Jahren halbieren das Risiko, an kolorektalem Karzinom zu erkranken, und senken die Gesamtmortalität um 65\% [54,55]. Wegen des häufig flachen Wachstums der Adenome ist die Schlingenabtragung jedoch nicht immer möglich, woraus die Notwendigkeit der chirurgischen Resektion resultiert. Treten multiple kolorektale Adenome synchron auf, ist die Kolektomie, gegebenenfalls die restaurative Proktokolektomie, zu erwägen.

\section{Resektion des manifesten kolorektalen Karzinoms} entsprechend den onkologischen Resektionsprinzipien Der etablierte Standard der chirurgischen Therapie des kolorektalen Karzinoms unter kurativer Zielsetzung besteht in der En-bloc-R0-Resektion des tumortragenden Kolons bzw. Rektums mit dem regionalen Lymphabflussgebiet nach onkologischen Radikalitätsprinzipien [56-58] und ist prinzipiell in dieser Form auch bei Patienten mit HNPCC gültig.

\section{Erweiterte Resektion bei manifestem kolorektalem Karzinom}

Grundlage für die Indikationsstellung zur Erweiterung des Eingriffs bei manifestem kolorektalem Karzinom sind das Risiko metachroner kolorektaler Karzinome von $40-45 \%$ in einem Zeitraum von 10 Jahren, die beschleunigte AdenomKarzinom-Sequenz und die rasche Tumorprogression [2, 51, 52]. Bei Erstmanifestation eines Karzinoms im Kolon erscheint daher eine subtotale Kolektomie indiziert. Bei Erstmanifestation eines Rektumkarzinoms sind eine Proktokolektomie bzw. unter entsprechenden onkologischen und funktionellen Voraussetzungen eine restaurative Prokokolektomie zu erwägen [27, 29, 59-63]. Da gegenwärtig jedoch keinerlei Daten prospektiver Studien zu diesem Thema existieren, besteht die Notwendigkeit der Evaluierung dieser Überlegungen im Rahmen einer prospektiven Studie.

Prophylaktische Resektion bereits vor der Manifestation eines kolorektalen Tumors

Für eine prophylaktischen Resektion (subtotale Kolektomie) vor der Entwicklung eines kolorektalen Tumors spricht das hohe kumulative Risiko von über $80 \%$ für ein kolorektales Karzinom [48, 49]. Hinzu kommt, dass trotz regelmäßiger Koloskopien bis zu 45\% der Mutationsträger ein kolorektales Karzinom entwickeln und etwa 8,7\% der Patienten an ihrer Tumorerkrankung sterben werden [54].

Gegen eine prophylaktische Kolektomie spricht die Penetranz von etwa $80 \%$, sodass etwa $20 \%$ der Mutationsträger umsonst kolektomiert werden würden. Hinzu kommt das Risiko für die Entwicklung der verschiedenen extrakolonischen Karzinome, deren chirurgische Prävention noch weitaus problematischer wäre. Eine prophylaktische Kolektomie kann gegenwärtig somit nicht empfohlen werden [48, 61]. Eine retrospektive Analyse kommt darüber hinaus zu dem Schluss, dass 
prophylaktische Operationen keinen signifikanten Überlebensvorteil gegenüber der koloskopischen Überwachung haben [64].

\section{Schlussfolgerung und Ausblick}

Kolorektale Adenome und Karzinome stellen eine der häufigsten Indikationen für endoskopische, minimal-invasive und chirurgische Interventionen im Bereich des Kolorektums dar. Die Bedeutung der frühzeitigen Diagnose und Sanierung von Adenomen resultiert aus der onkologischen Relevanz der Adenom-Karzinom-Sequenz sowohl bei den sporadischen als auch insbesondere, aufgrund der beschleunigten AdenomKarzinom Sequenz bzw. der deutlichen erhöhten Malignisierungsrate, bei den familiären Formen. Die technische Entwicklung der letzten Jahre hat unter Beachtung der entsprechenden Indikationsstellung eine Verringerung der chirurgischen Resektionsrate von kolorektalen Adenomen und
Frühkarzinomen bei gleicher Prognose und Komplikationsrate zugunsten minimal-invasiver Verfahren ermöglicht. Auf dem Gebiet der familiären Formen des kolorektalen Karzinoms hat die Einführung der molekularen Diagnostik zu einer verbesserten Prädiktion und Konzentration der notwendigen, engmaschigen Vorsorgeuntersuchungen auf Hochrisikopersonen (Mutationsträger) geführt.

Während auf dem Gebiet der konventionellen chirurgischen Resektionen keine wesentlich neuen Ergebnisse bezüglich der Prognose zu erwarten sind, liegen die abschließenden Ergebnisse im Sinne «harter Daten» für laparoskopische Resektionen bislang noch nicht vor, wenngleich bereits erste Studien gute Ergebnisse dazu aufwiesen.

Zuversichtlich erscheinen ebenfalls die vorläufigen Daten von Chemopräventionsstudien; dies könnte insbesondere bei Hochrisikopersonen einen neuen prophylaktischen Ansatz darstellen.

Kolorektalen Adenomen und Karzinomen wird auch zukünftig eine nicht unerhebliche Relevanz bezüglich Prophylaxe, Prädiktion, Diagnostik und Therapie zukommen.

\section{Literatur}

1 Fearon E R, Vogelstein B: A genetic model for colorectal tumorigenesis. Cell 1990;61:759-767.

2 Jass JR: Colorectal adenoma progression and genetic change: Is there a link? Ann Med 1995;27:301306.

3 Kinzler KW, Vogelstein B: Lessons from hereditary colorectal cancer. Cell 1996;87:159-170.

4 Kinzler KW, Vogelstein B: Cancer-susceptibility genes. Gatekeepers and caretakers. Nature 1997; 386:761-763.

5 Brandimarte G, Tursi A: Endoscopic snare excision of large pedunculated colorectal polyps: A new, safe, and effective technique. Endoscopy 2001;33 854-857.

6 Frühmorgen P, Demling L: Complications of diagnostic and therapeutic colonoscopy in the Federal Republic of Germany. Results of an inquiry. Endoscopy 1979;11:146-150.

7 Hackelsberger A, Frühmorgen P, Weiler H, Heller T, Seeliger H, Junghanns K: Endoscopic polypectomy and management of colorectal adenomas with invasive carcinoma. Endoscopy 1995;27:153-158.

8 Nusko G, Mansmann U, Partzsch U, AltendorfHofmann A, Groitl H, Wittekind C, Ell C, Hahn EG: Invasive carcinoma in colorectal adenomas: Multivariate analysis of patient and adenoma characteristics. Endoscopy 1997;29:626-631.

9 Nivatvongs S: Surgical management of early colorectal cancer. World J Surg 2000;24:1052-1055.

10 Mellgren A, Sirivongs P, Rothenberger DA, Madoff RD, Garcia-Aguilar J: Is local excision adequate therapy for early rectal cancer? Dis Colon Rectum 2000;43:1064-1071; discussion 71-74.

11 Winde G, Nottberg H, Keller R, Schmid K W, Bunte H: Surgical cure for early rectal carcinomas (T1). Transanal endoscopic microsurgery vs. anterior resection. Dis Colon Rectum 1996;39:969-976.

12 Heintz A, Morschel M, Junginger T: Comparison of results after transanal endoscopic microsurgery and radical resection for $\mathrm{T} 1$ carcinoma of the rectum. Surg Endosc 1998;12:1145-1148.
13 Morschel M, Heintz A, Bussmann M, Junginger T: Follow-up after transanal endoscopic microsurgery or transanal excision of large benign rectal polyps. Langenbecks Arch Surg 1998;383:320-324.

14 Bohm B, Schwenk W, Muller JM: Long-term results after laparoscopic resection of colorectal carcinoma. Chirurg 1999;70:453-455.

15 Hartley JE, Mehigan BJ, MacDonald AW, Lee PW, Monson JR: Patterns of recurrence and survival after laparoscopic and conventional resections for colorectal carcinoma. Ann Surg 2000;232:181-816.

16 Kockerling F, Rose J, Schneider C, Scheidbach H, Scheuerlein H, Reymond MA, Reck T, Konradt J, Bruch HP, Zornig C, Barlehner E, Kuthe A, Szinicz G, Richter HA, Hohenberger W: Laparoscopic colorectal anastomosis: Risk of postoperative leakage. Results of a multicenter study. Laparoscopic Colorectal Surgery Study Group (LCSSG). Surg Endosc 1999;13:639-644.

17 Köckerling F, Scheidbach H, Schneider C, Barlehner E, Kohler L, Bruch H P, Konradt J, Wittekind C, Hohenberger W: Laparoscopic abdominoperineal resection: Early postoperative results of a prospective study involving 116 patients. The Laparoscopic Colorectal Surgery Study Group. Dis Colon Rectum 2000;43:1503-1511.

18 Pistorius S, Schackert HK, Saeger H-D: Can molecular knowledge from studies of hereditary carcinoma applied to sporadic colorectal carcinoma? Kongressbd Dtsch Ges Chir Kongr 2001; 118:820824

19 Hawk E, Lubet R, Limburg P: Chemoprevention in hereditary colorectal cancer syndromes. Cancer 1999;86:2551-2563.

20 Steinbach G, Lynch PM, Phillips RK, Wallace M H, Hawk E, Gordon GB, Wakabayashi N, Saunders B, Shen Y, Fujimura T, Su L K, Levin B: The effect of celecoxib, a cyclooxygenase-2 inhibitor, in familial adenomatous polyposis. N Engl J Med 2000;342: 1946-1952.
21 Ruschoff J, Wallinger S, Dietmaier W, Bocker T, Brockhoff G, Hofstadter F, Fishel R: Aspirin suppresses the mutator phenotype associated with hereditary nonpolyposis colorectal cancer by genetic selection. Proc Natl Acad Sci U S A 1998;95: 11301-11306

22 Garcia-Rodriguez LA, Huerta-Alvarez C: Reduced risk of colorectal cancer among long-term users of aspirin and nonaspirin nonsteroidal antiinflammatory drugs. Epidemiology 2001;12:88-93.

23 Nagase H, Miyoshi Y, Horii A, Aoki T, Ogawa M, Utsunomiya J, Baba S, Sasazuki T, Nakamura Y: Correlation between the location of germ-line mutations in the APC gene and the number of colorectal polyps in familial adenomatous polyposis patients. Cancer Res 1992;52:4055-4057.

24 Caspari R, Friedl W, Mandl M, Moslein G, Kadmon M, Knapp M, Jacobasch KH, Ecker KW, KreisslerHaag D, Timmermanns G: Familial adenomatous polyposis: Mutation at codon 1309 and early onset of colon cancer. Lancet 1994;343:629-632.

25 Friedl W, Meuschel S, Caspari R, Lamberti C, Krieger S, Sengteller M, Propping P: Attenuated familial adenomatous polyposis due to a mutation in the 3 ' part of the APC gene. A clue for understanding the function of the APC protein. Hum Genet 1996; 97:579-584.

26 Winawer SJ, Fletcher RH, Miller L, Godlee F, Stolar M H, Mulrow CD, Woolf SH, Glick SN, Ganiats T G, Bond JH, Rosen L, Zapka JG, Olsen SJ, Giardiello FM, Sisk JE, van Antwerp R, Brown-Davis C, Marciniak DA, Mayer RJ: Colorectal cancer screening: Clinical guidelines and rationale. Gastroenterology 1997;112:594-642.

27 Vasen HF: Clinical diagnosis and management of hereditary colorectal cancer syndromes. J Clin Oncol 2000;18:81S-92S.

28 Vasen HF: When should endoscopic screening in familial adenomatous polyposis be started? Gastroenterology 2000;118:808-809. 
29 Soravia C, Berk T, Cohen Z: Genetic testing and surgical decision making in hereditary colorectal cancer. Int J Colorectal Dis 2000;15:21-28.

30 Vasen HF, van der Luijt RB, Slors JF, Buskens E, de Ruiter P, Baeten CG, Schouten WR, Oostvogel HJ, Kuijpers JH, Tops CM, Meera Khan P: Molecular genetic tests as a guide to surgical management of familial adenomatous polyposis. Lancet 1996; 348:433-435.

31 Wu JS, Paul P, Mcgannon EA, Church JM: APC genotype, polyp number, and surgical options in familial adenomatous polyposis. Ann Surg 1998;227: 57-62.

32 Choi HS, Park YJ, Youk EG, Yoon KA, Ku JL, Kim NK, Kim SM, Kim YJ, Moon DJ, Min JS, Park CJ, Bae OS, Yang DH, Jun SH, Chung ES, Jung PM, Whang Y, Park JG: Clinical characteristics of Peutz-Jeghers syndrome in Korean polyposis patients. Int J Colorectal Dis 2000;15:35-38.

33 Wang ZJ, Ellis I, Zauber P, Iwama T, Marchese C, Talbot I, Xue WH, Yan ZY, Tomlinson I: Allelic imbalance at the LKB1 (STK11) locus in tumours from patients with Peutz-Jeghers' syndrome provides evidence for a hamartoma-(adenoma)-carcinoma sequence. J Pathol 1999;188:9-13.

34 Gruber SB, Entius MM, Petersen GM, Laken SJ, Longo PA, Boyer R, Levin M, Mujumdar U J, Trent J M, Kinzler K W, Vogelstein B, Hamilton S R, Polymeropoulos MH, Offerhaus GJ, Giardiello FM: Pathogenesis of adenocarcinoma in Peutz-Jeghers syndrome. Cancer Res 1998;58:5267-5270.

35 Spigelman AD, Arese P, Phillips R K: Polyposis: The Peutz-Jeghers syndrome. Br J Surg 1995;82: 1311-1314.

36 Giardiello FM, Welsh SB, Hamilton SR, Offerhaus GJ, Gittelsohn M, Booker SV, Krush J, Yardley JH, Luk GD: Increased risk of cancer in the Peutz-Jeghers syndrome. N Engl J Med 1987;316:1511-1514.

37 Spigelman AD, Murday V, Phillips RK: Cancer and the Peutz-Jeghers syndrome. Gut 1989;30:15881590 .

38 Boardman LA, Thibodeau SN, Schaid DJ, Lindor NM, McDonnell SK, Burgart LJ, Ahlquist DA, Podratz KC, Pittelkow M, Hartmann LC: Increased risk for cancer in patients with the Peutz-Jeghers syndrome. Ann Intern Med 1998;128:896-899.

39 Tomlinson IP, Houlston RS: Peutz-Jeghers syndrome. J Med Genet 1997;34:1007-1011.

40 Ishida H, Murata N, Tada M, Takada S, Fujioka M, Idezuki Y: A new simple technique for performing intraoperative endoscopic resection of small-bowel polyps in patients with Peutz-Jeghers syndrome. Surg Today 1999;29:581-583.

41 Taira K, Matsubara H, Isa T, Miyazato H, Hiroyasu S, Shiraishi M, Muto Y, Kinjo F: Combined endoscopic and surgical treatment for multiple polyps of the small intestine in Peutz-Jeghers syndrome: A case report. Surg Laparosc Endosc Percutan Tech 2000;10:409-411.

42 Lin BC, Lien JM, Chen RJ, Fang JF, Wong YC: Combined endoscopic and surgical treatment for the polyposis of Peutz- Jeghers syndrome. Surg Endosc 2000;14:1185-1187.

43 Jass JR, Williams CB, Bussey HJ, Morson BC: Juvenile polyposis - a precancerous condition. Histopathology 1988;13:619-630.

44 Howe JR, Mitros FA, Summers RW: The risk of gastrointestinal carcinoma in familial juvenile polyposis. Ann Surg Oncol 1998;5:751-756.

45 Giardiello FM, Hamilton SR, Kern SE, Offerhaus GJ, Green PA, Celano P, Krush AJ, Booker SV: Colorectal neoplasia in juvenile polyposis or juvenile polyps. Arch Dis Child 1991;66:971-975.
46 Jarvinen H, Franssila KO: Familial juvenile polyposis coli; increased risk of colorectal cancer. Gut 1984;25:792-800

47 Scott-Conner CE, Hausmann M, Hall TJ, Skelton DS, Anglin BL, Subramony C: Familial juvenile polyposis: Patterns of recurrence and implications for surgical management. J Am Coll Surg 1995;181: 407-413.

48 Vasen HF, Wijnen JT, Menko FH, Kleibeuker JH, Taal BG, Griffioen G, Nagengast FM, Meijers-Heijboer EH, Bertario L, Varesco L, Bisgaard ML, Mohr J, Fodde R, Khan PM: Cancer risk in families with hereditary nonpolyposis colorectal cancer diagnosed by mutation analysis. Gastroenterology 1996;110:1020-1027.

49 Dunlop MG, Farrington SM, Carothers AD, Wyllie AH, Sharp L, Burn J, Liu B, Kinzler KW, Vogelstein B: Cancer risk associated with germline DNA mismatch repair gene mutations. Hum Mol Genet 1997;6:105-110.

50 Aarnio M, Sankila R, Pukkala E, Salovaara R, Aaltonen LA, De La Chapelle A, Peltomaki P, Mecklin JP, Jarvinen HJ: Cancer risk in mutation carrier of DNA-mismatch-repair genes. Int J Cancer 1999; 81:214-218.

51 Aarnio M, Mecklin JP, Aaltonen LA, NystromLahti M, Jarvinen HJ: Life-time risk of different cancers in hereditary non-polyposis colorectal cancer (HNPCC) syndrome. Int J Cancer 1995;64:430 433.

52 Lynch HT: Is there a role for prophylactic subtota colectomy among hereditary nonpolyposis colorec tal cancer germline mutation carriers? Dis Colon Rectum 1996;39:109-110.

53 Burke W, Daly M, Garber J, Botkin J, Kahn MJ, Lynch P, Mctiernan A, Offit K, Perlman J, Petersen G, Thomson E, Varricchio C: Recommendations for follow-up care of individuals with an inherited predisposition to cancer. II. BRCA1 and BRCA2. Cancer Genetics Studies Consortium. Jama 1997; 277:997-1003.

54 Jarvinen HJ, Mecklin JP, Sistonen P: Screening reduces colorectal cancer rate in families with hereditary nonpolyposis colorectal cancer. Gastroenterology 1995;108:1405-1411.

55 Jarvinen HJ, Aarnio M, Mustonen H, Aktan-Collan K, Aaltonen LA, Peltomaki P, De La Chapelle A, Mecklin JP: Controlled 15-year trial on screening for colorectal cancer in families with hereditary nonpolyposis colorectal cancer. Gastroenterology 2000;118:829-834.

56 Junginger T, Mentges B: Principles of radical treatment in surgery of colonic cancer. Langenbecks Arch Chir 1987;372:481-486.

57 Lehnert T, Herfarth C: Principles and value of lymph node excision in colorectal carcinoma. Chirurg 1996;67:889-899.

58 Herfarth C, Runkel N: Surgical standards in primary colon cancer. Chirurg 1994;65:514-523.

59 Box JC, Rodriguez-Bigas MA, Weber TK, Petrelli NJ: Clinical implications of multiple colorectal carcinomas in hereditary nonpolyposis colorectal carcinoma. Dis Colon Rectum 1999;42:717-721.

60 Church JM: Prophylactic colectomy in patients with hereditary nonpolyposis colorectal cancer. Ann Med 1996;28:479-482.

61 Pistorius SR, Nagel M, Kruger S, Plaschke J, Kruppa C, Wehrmann U, Schackert HK, Saeger H-D: Combined molecular and clinical approach for decision making for surgery in HNPCC patients: A report on three cases in two families. Int J Colorectal Dis 2001;16:402-407.
62 Rodriguez-Bigas MA, Vasen HF, Pekka-Mecklin J, Myrhoj T, Rozen P, Bertario L, Jarvinen HJ, Jas JR, Kunitomo K, Nomizu T, Driscoll DL: Rectal cancer risk in hereditary nonpolyposis colorectal cancer after abdominal colectomy. Internationa Collaborative Group on HNPCC. Ann Surg 1997; 225:202-207.

63 Schackert HK, Hahn M, Pistorius S, Saeger H-D Preventive surgery of inherited colorectal cancer identified through molecular diagnosis. Chirurg 1999;70:345-352.

64 Syngal S, Weeks J C, Schrag D, Garber JE, Kuntz KM: Benefits of colonoscopic surveillance and prophylactic colectomy in patients with hereditary nonpolyposis colorectal cancer mutations. Ann Intern Med 1998;129:787-796.

65 Groden J, Thliveris A, Samowitz W, Carlson M, Gelbert L, Albertsen H, Joslyn G, Stevens J, Spirio L, Robertson M: Identification and characterization of the familial adenomatous polyposis coli gene. Cell 1991;66:589-600.

66 Joslyn G, Carlson M, Thliveris A, Albertsen H, Gelbert L, Samowitz W, Groden J, Stevens J, Spirio L, Robertson M,et al: Identification of deletion mutations and three new genes at the familial polyposis locus. Cell 1991;66:601-613.

67 Kinzler KW, Nilbert MC, Su LK, Vogelstein B, Bryan TM, Levy DB, Smith K, Preisinger AC, Hedge P, Mckechnie D, et al: Identification of FAP locus genes from chromosome 5q21. Science 1991 253:661-665.

68 Nakamura Y, Nishisho I, Kinzler KW, Vogelstein B, Miyoshi Y, Miki Y, Ando H, Horii A, Nagase H: Mutations of the adenomatous polyposis coli gene in familial polyposis coli patients and sporadic colorectal tumors. Princess Takamatsu Symp 1991;22: 285-292.

69 Jenne D E, Reimann H, Nezu J, Friedel W, Loff S, Jeschke R, Muller O, Back W, Zimmer M: Peutz Jeghers syndrome is caused by mutations in a novel serine threonine kinase. Nat Genet 1998;18:38-43.

70 Nakagawa H, Koyama K, Miyoshi Y, Ando H, Baba S, Watatani M, Yasutomi M, Matsuura N, Monden M, Nakamura Y: Nine novel germline mutations of STK11 in ten families with Peutz- Jeghers syndrome. Hum Genet 1998;103:168-172.

71 Resta N, Simone C, Mareni C, Montera M, Gentile M, Susca F, Gristina R, Pozzi S, Bertario L, Bufo P, Carlomagno N, Ingrosso M, Rossini FP, Tenconi R, Guanti G: STK11 mutations in Peutz-Jeghers syndrome and sporadic colon cancer. Cancer Res 1998; 58:4799-801.

72 Howe JR, Roth S, Ringold JC, Summers RW, Jarvinen HJ, Sistonen P, Tomlinson IP, Houlston RS, Bevan S, Mitros FA, Stone EM, Aaltonen LA: Mutations in the SMAD4/DPC4 gene in juvenile polyposis. Science 1998;280:1086-1088.

73 Howe JR, Bair JL, Sayed MG, Anderson ME, Mitros FA, Petersen GM, Velculescu VE, Traverso G, Vogelstein B: Germline mutations of the gene encoding bone morphogenetic protein receptor $1 \mathrm{~A}$ in juvenile polyposis. Nat Genet 2001;28:184-187.

74 Zhou XP, Woodford-Richens K, Lehtonen R, Kurose K, Aldred M, Hampel H, Launonen V, Virta S, Pilarski R, Salovaara R, Bodmer WF, Conrad BA, Dunlop M, Hodgson SV, Iwama T, Jarvinen H, Kellokumpu I, Kim JC, Leggett B, Markie D, Mecklin J P, Neale K, Phillips R, Piris J, Rozen P, Houlston RS, Aaltonen LA, Tomlinson IP, Eng C: Germline mutations in BMPR1A/ALK3 cause a subset of cases of juvenile polyposis syndrome and of Cowden and Bannayan-Riley-Ruvalcaba syndromes. Am J Hum Genet 2001;69:704-711. 
75 Kolodner RD, Hall NR, Lipford J, Kane MF, Morrison PT, Finan PJ, Burn J, Chapman P, Earabino C, Merchant E, et al: Structure of the human MLH1 locus and analysis of a large hereditary nonpolyposis colorectal carcinoma kindred for mlh1 mutations. Cancer Res 1995;55:242-248.

76 Leach FS, Nicolaides NC, Papadopoulos N, Liu B, Jen J, Parsons R, Peltomaki P, Sistonen P, Aaltonen LA, Nystrom-Lahti M, et al: Mutations of a mutS homolog in hereditary nonpolyposis colorectal cancer. Cell 1993;75:1215-1225.

77 Akiyama Y, Sato H, Yamada T, Nagasaki H, Tsuchiya A, Abe R, Yuasa Y: Germ-line mutation of the hMSH6/GTBP gene in an atypical hereditary nonpolyposis colorectal cancer kindred. Cancer Res 1997;57:3920-3923.

78 Miyaki M, Konishi M, Tanaka K, Kikuchi-Yanoshita R, Muraoka M, Yasuno M, Igari T, Koike M, Chiba M, Mori T: Germline mutation of MSH6 as the cause of hereditary nonpolyposis colorectal cancer. Nat Genet 1997;17:271-272.
79 Nicolaides NC, Papadopoulos N, Liu B, Wei YF, Carter KC, Ruben SM, Rosen CA, Haseltine WA, Fleischmann RD, Fraser CM, et al: Mutations of two PMS homologues in hereditary nonpolyposis colon cancer. Nature 1994;371:75-80.

80 Papadopoulos N, Nicolaides NC, Wei YF, Ruben SM, Carter KC, Rosen CA, Haseltine WA, Fleischmann RD, Fraser CM, Adams MD, et al: Mutation of a mutL homolog in hereditary colon cancer. Science 1994;263:1625-1629.

81 Plaschke J, Kruppa C, Tischler R, Bocker T, Pistorius S, Dralle H, Ruschoff J, Saeger H-D, Fishel R, Schackert HK: Sequence analysis of the mismatch repair gene hMSH6 in the germline of patients with familial and sporadic colorectal cancer. Int J Cancer 2000;85:606-613.
82 Wu Y, Berends MJ, Post JG, Mensink RG, Verlind E, van der Sluis T, Kempinga C, Sijmons RH, van der Zee AG, Hollema H, Kleibeuker JH, Buys CH, Hofstra RM: Germline mutations of EXO1 gene in patients with hereditary nonpolyposis colorectal cancer (HNPCC) and atypical HNPCC forms. Gastroenterology 2001;120:1580-1587.

83 Vasen HF, Mecklin JP, Khan PM, Lynch HT: The International Collaborative Group on Hereditary Non-Polyposis Colorectal Cancer (ICG-HNPCC). Dis Colon Rectum 1991;34:424-425.

84 Vasen HF, Watson P, Mecklin JP, Lynch HT: New clinical criteria for hereditary nonpolyposis colorectal cancer (HNPCC, Lynch syndrome) proposed by the International Collaborative group on HNPCC. Gastroenterology 1999;116:1453-1456.

\title{
Ludwigshafener Gastroenterologische Tage
}

vom 28.-30. November 2002

\author{
Tagungspräsident: Prof. Dr. J. F. Riemann \\ Kongress-Sekretäre: PD Dr. H. E. Adamek, Dr. U. Weikert
}

\section{Themen:}

28.11.2002 / 9:00-18:30 Uhr

29.11.2002 / 9:00-18:30 Uhr

30.11.2002 / 9:00-13:00 Uhr
Laparoskopietag - Klinikum Ludwigshafen

Internationales Symposium

„Fortschritte in der Endoskopie“ Pfalzbau, Ludwigshafen

Live-Demonstrationen aus der neuen Endoskopieabteilung des Klinikums Ludwigshafen

Gastroenterologisches Forum - Pfalzbau, Ludwigshafen

\section{Auskunft und Anmeldung:}

Frau Gaby Kneissler

Kongress-Sekretariat der Med. Klinik C

Klinikum Ludwigshafen gGmbH

Bremserstr. 79, 67063 Ludwigshafen

Tel.: +49 (0)621-503 4111, Fax: -4112

kongress-sekretariat-medc@t-online.de 